\title{
Models for Type Ia supernovae and related astrophysical transients
}

\author{
Friedrich K. Röpke · Stuart A. Sim
}

Received: date / Accepted: date

\begin{abstract}
We give an overview of recent efforts to model Type Ia supernovae and related astrophysical transients resulting from thermonuclear explosions in white dwarfs. In particular we point out the challenges resulting from the multi-physics multi-scale nature of the problem and discuss possible numerical approaches to meet them in hydrodynamical explosion simulations and radiative transfer modeling. We give examples of how these methods are applied to several explosion scenarios that have been proposed to explain distinct subsets or, in some cases, the majority of the observed events. In case we comment on some of the successes and shortcoming of these scenarios and highlight important outstanding issues.
\end{abstract}

\section{Introduction}

The theoretical description of Type Ia supernovae and related astrophysical transients as thermonuclear explosions of white dwarfs stars has seen rapid development over the past decade. Multidimensional hydrodynamical simulations of the explosion phase were conducted, and the results could be directly used as input for radiative transfer simulations that derive synthetic observables from such models in a consistent way. This allowed to connect modern supernova theory directly to astronomical observations and facilitated a way to validate modeling assumptions by comparison with astronomical data.

\section{F. K. Röpke}

Zentrum für Astronomie der Universität Heidelberg, Philosophenweg 12, 69120 Heidelberg, Germany

and

Heidelberger Institut für Theoretische Studien, Schloss-Wolfsbrunnenweg 35, 69118

Heidelberg, Germany

E-mail: friedrich.roepke@h-its.org

S. A. Sim

School of Mathematics and Physics, Queen's University Belfast, Belfast BT7 1NN, UK 
The result of theoretical efforts is a consistent theoretical modeling pipeline for thermonuclear explosions in white dwarf stars. It starts out from a model of the progenitor and extends over multidimensional hydrodynamical simulations of the explosion phase. Nucleosynthesis processes in it are usually determined in a post-processing step. This gives a multidimensional picture of the structure (in particular the density, the velocity and the chemical composition) of the ejecta cloud, that serves as an input to radiative transfer calculations. These, in turn, allow to derive synthetic observables.

In the two parts of this article, we discuss two main ingredients to this modeling pipeline: hydrodynamics simulations of the explosion phase together with nucleosynthesis calculations, and the radiative transfer in the ejecta.

\section{Explosion modeling}

\subsection{Ansatz and scale challenges}

The progenitor star of a thermonuclear supernova event is a macroscopic object; densities are high and the typical spatial scales of interest are large. Therefore, the modeling ansatz is based on the equations of fluid dynamics, specifically the Euler equations describing ideal fluids. Viscosity effects are sub-dominant on the scales considered in the model and numerical viscosity in any case outweighs physical effects.

As nuclear burning powers the supernova explosion, reactions have to be taken into account. In addition to the usual fluid dynamics equations describing mass conservation, momentum, and energy balance, a set of equations is necessary to capture species balance. Source terms account for species conversion and the associated energy release. For a complete description of thermonuclear combustion processes, several other effects, such as heat conduction, have to be included in the model (see, e.g., Röpke 2017 for a recent overview). The solution of this system of equations in a numerical supernova simulation, however, is not straightforward because of massive challenges arising from the extremely wide range of relevant scales.

For a numerical treatment, the underlying system of equations is discretized, most commonly in a finite-volume approach. The supernova explosion takes place on the order of the dynamical time scale and therefore time discretization usually follows an explicit scheme. Such schemes are only conditionally stable and consequently the numerical time step has to be restricted according to the Courant-Friedrichs-Lewi (CFL) condition, which, in loose terms, requires numerical time steps to be taken smaller than the sound crossing time over a computational grid cell. The sound crossing time over an entire WD star is on the order of a second; hence the numerical time steps in supernova simulations stay far below a second. The evolution time scale of the progenitor, in contrast, is set by nuclear burning, that lasts many orders of magnitude longer than this. Clearly, this phase is not accessible to multidimensional hydrodynamic simulations. Depending on the propagation mode of the resulting thermonuclear combustion wave, 
the ignition itself may take centuries or happen dynamically. Thus, ignition is a marginal case that may be addressed in the framework of hydrodynamical simulations, at least as much as time scales are concerned. The explosion itself proceeds in the transonic regime and is certainly accessible to such a numerical treatment.

The time scales on which most observables form are much longer days, weeks, or months. Because the supernova ejecta are in homologous expansion by then (hydrodynamical effects are frozen out) and the radiation field is dynamically unimportant (at least to zeroth order; see Woosley et al. 2007 for a discussion of the effect of ${ }^{56} \mathrm{Ni}$ decay on the density and velocity profiles), this can be treated in a modeling approach that is separated from the hydrodynamical simulations of the explosion phase and uses their results only to define the background state of the expanding ejecta (see Sect. 3).

The spatial scale problem in thermonuclear supernova models is no less challenging. Due to the extreme temperature sensitivity of the involved nuclear reactions, burning is confined to the hottest regions and propagates in thin fronts. Typically, these have widths far below the millimeter scale. This scale is extremely small compared with that of the exploding white dwarfs (with radii of a few thousand kilometers). Seen from the large global scales, it is well-justified to approximate combustion waves as sharp discontinuities separating the fuel from the ash material. In this discontinuity approximation, jump conditions over the combustion front can be established according to the laws of fluid dynamics. They distinguish between two modes of propagation for the combustion front: subsonic deflagration and supersonic detonation.

Both deflagrations and detonations are subject to multidimensional hydrodynamic instabilities (for a recent review see Röpke 2017). While for the latter case, it is generally assumed that the effects on the overall explosion process are weak, deflagration burning is most likely dominated and as a consequence significantly boosted - by the interaction with such instabilities. If ignited near the center of the white dwarf star, a deflagration becomes turbulent. This is an implication of buoyancy instability between the central hot and light ashes and the dense and cold unburnt fuel ahead of the flame. As a result, in the non-linear regime of the LandauRayleigh-Taylor instability, bubbles of burning material rise towards the stellar surface (but see Hristov et al. 2017). The flame front is located at their interfaces. Outside of the bubbles, cold unburnt material sinks down towards the center of the white dwarf. This leads to shear motions at the flame. Typical Reynolds numbers are as high as $10^{14}$ and consequently a turbulent energy cascade forms. At the largest scales, kinetic energy is injected by large-scale turbulent eddies, that subsequently decay to smaller scales constituting the inertial range, in which kinetic energy is transported from the large to the small scales without energy loss. Only at the microscopic Kolmogorov scale, the turbulent energy is finally converted to heat by viscous effects.

On a wide sub-range of that turbulent cascade, the deflagration flame interacts with turbulent eddies (see Röpke and Schmidt 2009 and Röpke 2017 for discussions of turbulent deflagrations in SNe Ia). The effect of this 
interaction depends on whether turbulence corrugates the flame structure only on large scales, or whether it penetrates the internal flame structure and modifies the microphysical transport in it. The first case, which corresponds to the so-called flamelet regime of turbulent combustion, applies to most of the explosion period. Here, the flame front is stretched out and wrinkled so that its surface area is greatly enlarged.

Only at the latest times, when the star has expanded significantly and the burning densities are low, the flame structure broadens. With the expansion, turbulence gradually freezes out, but if the prevailing turbulent intensities are still high, a modification of the flame structure is expected. It has been suggested (e.g. Khokhlov et al. 1997, Lisewski et al.|2000a, Röpke et al. 2007, Woosley 2007, Schmidt et al.2010, Poludnenko et al. 2011) that in this regime transitions of the flame propagation mode from subsonic deflagration to supersonic detonation are possible. Such deflagration-to-detonation transitions (DDTs) are observed in terrestrial chemical combustion, but there they are mostly associated with obstacles or walls of the combustion vessel. The existence of unconfined DDTs, as would be required in the astrophysical context, remains unproven. Sufficiently strong turbulent mixing inside a broad flame structure is proposed to lead to conditions in which a detonation wave can form via the Zel'dovich gradient mechanism (Zel'dovich et al. 1970).

In addition to these uncertainties in the flame propagation mechanism, the problem of the initial conditions poses a fundamental challenge to modeling thermonuclear supernova explosions. As to now, progenitor systems of Type Ia supernovae are not observationally established. Although the astronomical identification of a progenitor would help to constrain potential scenarios, it would not completely solve all problems of initial conditions for explosion simulations. The progenitor structure and the ignition process are not directly accessible to observations and have to be modeled. As discussed above, the timescales dominating the pre-ignition evolution phases cannot easily be addressed in multidimensional simulations. The resulting uncertainty in the initial conditions is a fundamental obstacle to explosion modeling. The equations of hydrodynamics forming the basis for the description of the explosion processes are hyperbolic partial differential equations. Thus they pose initial value problems. The choice of the initial conditions therefore has a strong impact on the numerical solution (or even determines it). One should thus avoid to draw conclusions from thermonuclear supernova simulations that are dominated by an arbitrary of the initial conditions.

\subsection{Numerical implementation}

Several approaches have been taken by different groups to meet the challenges laid out above and perform simulations of thermonuclear supernova explosions. An overview of modeling the combustion physics is given in Röpke (2017). Here, we will focus on one particular choice.

The impracticality to resolve the tiny internal structure of combustion waves in full-star supernova explosion simulations requires to model their 
propagation in parametrized approaches. The physical structure is either artificially broadened so that it can be represented on the computational grid (Khokhlov 1995, Vladimirova et al. 2006, Calder et al. 2007), or it is completely ignored and the combustion front is treated as a sharp discontinuity separating the fuel from the ashes. An appropriate technique to achieve this (at least up to the spread in hydrodynamical quantities introduced by the numerical Riemann solver) is the so-called level-set scheme (Osher and Sethian 1988, Reinecke et al. 1999). In this front-tracking method, the combustion wave is associated with the zero level-set of a signed distance function $G$. Its motion is due to advection of the $G$-field and due to burning. This is captured by an appropriate "level-set equation". While the advection part can be determined from the underlying hydrodynamics scheme, the advancement due to burning is not consistently treated in the discontinuity approximation. It is a parameter of the model that has to be determined externally. For laminar deflagration flames, for instance, it can be derived from resolved one-dimensional simulations (e.g. Timmes and Woosley 1992). For detonations, the Chapman-Jouguet case is a reasonable approximation at low fuel densities. At higher densities, however, nuclear statistical equilibrium establishes behind the detonation front and reactions are partially endothermic. This gives rise to detonations of "pathological" type, that have to be studied in off-line simulations (Sharpe 1999).

The fundamental importance of hydrodynamical instabilities for the propagation of deflagrations requires special modeling approaches. As discussed in Sect. 2.1. the interaction of the flame front with self-generated turbulence boosts the burning efficiency. Because only the largest scales of the turbulent cascade are resolved, the effect of flame surface enlargement due to interaction with turbulent eddies on smaller scales has to be compensated by imposing an effective turbulent burning speed on the scale of numerical resolution. This effective turbulent flame propagation velocity replaces the laminar flame speed in the level-set equation. According to Damköhler (1940), it scales with the turbulent velocity fluctuations on the considered length scale. Because of numerical dissipation, these are difficult to determine close to the resolution of the computational grid, and therefore turbulent subgrid-scale models are employed to determine them (see e.g. Niemeyer and Hillebrandt 1995, Schmidt et al. 2006, Röpke and Schmidt 2009, Ciaraldi-Schoolmann et al. |2009, Hicks and Rosner 2013 , Jackson et al. 2014, Röpke 2017 for a discussion of approaches used in SN Ia explosion models). It is one of the important achievements of multidimensional simulations to capture the effect of turbulent flame acceleration in a self-consistent way.

Another challenge is the modeling of nuclear reactions that take place in and behind the combustion wave. Two major obstacles have to be overcome in this context. The first is that many reactions are involved in the burning and an extended nuclear network is necessary to predict the synthesis of all involved isotopes. Solving the full network concurrently with the hydrodynamic simulation requires substantial computational effort, in particular in three-dimensional setups. While this is a practical challenge, the second is more fundamental. If combustion waves are represented as 
discontinuities, their internal structure and details of the reactions are not captured. Artificially broadened combustion waves face the problem that the length scales on which the species conversion and energy release proceed physically are not resolved. They are also challenged by the numerical effort of an extended nuclear network. For this reason, reduced nuclear networks are usually employed in the hydrodynamic explosion simulations, that follow only a few representative species (accounting, for instance, for unburnt fuel material, intermediate mass elements, and nuclear statistical equilibrium compositions). The primary goal of the description of nuclear reactions in the hydrodynamic explosion simulations is to model the energy release driving the dynamics. With reduced networks and artificially broadened combustion waves, it is possible to approximate the energy release to a sufficient accuracy. In models with very few representative species and/or discontinuity descriptions of the combustion waves, the energy release cannot be consistently reproduced and has to be calibrated. This is either done on the basis of one-dimensional resolved flame simulations or in an iterative procedure involving a sequence of explosion models and nucleosynthesis post-processing step. Such post-processing is also necessary to achieve the secondary goal of modeling the burning processes: the determination of detailed nucleosynthetic yields and the chemical structure of ejected material in thermonuclear supernova explosions. The key idea is to place virtual particles (so-called tracers) in the material of the exploding white dwarf star so that each represents a certain fraction of the total mass. These tracer particles are then passively advected with the flow of the exploding material and record the thermodynamic trajectories representative for the fraction of mass they follow. This data is then used as input to a post-processing step that reconstructs the details of the nuclear reactions based on an extended nuclear reaction network (see, e.g., Travaglio et al. 2004 ).

The detailed hydrodynamic and chemical structure of the ejected material is part of a modeling pipeline that follows the supernova event from the progenitor structure over hydrodynamical explosion simulations and nucleosynthetic post-processing to the formation of observables that can then be compared to astronomical data. It is input to multidimensional radiative transfer calculations that will be discussed in Sect. 3.

\subsection{Requirements for a viable explosion scenario}

A fundamental goal of modeling thermonuclear explosions in white dwarfs is to reproduce the characteristic spectral features of Type Ia supernovae. The lack of hydrogen and helium is characteristic for this class of objects. Moreover, spectral features indicate the presence of substantial amounts of iron group and intermediate-mass elements. This is prototypical for burning carbon-oxygen white dwarf matter. Irrespective of the combustion wave being a deflagration or a detonation, the released energy and the composition of the ash depends on the fuel density ahead of the front.

At the highest densities, as encountered in the cores of massive white dwarf stars, the ash temperatures become high enough to establish nuclear 
statistical equilibrium (NSE) conditions. Freeze-out from NSE occurs when the ejecta expand and iron group nuclei are formed. At lower fuel densities, burning is incomplete and intermediate-mass elements $(\mathrm{Si}, \mathrm{S}, \mathrm{Ca}$, etc.) are synthesized. At even lower densities, carbon burns to oxygen, and below a certain threshold, burning ceases and unprocessed carbon-oxygen white dwarf material is left behind.

The fact that intermediate-mass elements are seen in the spectra implies that a substantial amount of the stellar material must be processed at sufficiently low densities ( $\rho_{\text {fuel }} \lesssim 10^{7} \mathrm{~g} \mathrm{~cm}^{-3}$ ) to enable incomplete burning. The burning front therefore must either (1) pre-expand a Chandrasekharmass WD, which requires a sub-sonic flame propagation mode, (2) proceed as a detonation in a pre-expanded Chandrasekhar-mass WD in a delayed detonation scenario, or (3) form a detonation in a sub-Chandrasekhar mass WD. We will discuss these possibilities in Sect. 4 .

\section{Prediction of observables}

\subsection{Radiative transfer considerations}

As outlined above, hydrodynamical explosion models can simulate the dynamics and nucleosynthesis in thermonuclear supernovae from the point of ignition until the ejecta reach near-homologous expansion. However, to assess the validity of such models, the explosion model output must be mapped onto the space of observable quantities that can be compared to data. In general, this requires additional calculations that yield predicted light curves, spectra and/or spectropolarimetry. Fortunately, as noted above, such calculations can usually be performed as a post-processing step on the ejecta that have been dynamically simulated into the homologous phase. There are important exceptions, however, most notably for scenarios that involve ongoing dynamics as the explosion ejecta interact with a dense environment (see, e.g., Fryer et al.|2010, Blinnikov and Sorokina|2010, Noebauer et al. 2016).

Thermonuclear supernovae have ejecta that are rich in heavy elements: in models of normal SNe Ia, around half of the ejected mass is composed of iron-peak elements, with around half of the remainder being the so-called intermediate-mass elements ( $\mathrm{Si}, \mathrm{S}, \mathrm{Ca}$ etc.). The relative complexity of the atomic structure of the first few ions of these elements (compared to e.g. $\mathrm{H}$ or $\mathrm{He}$, which are more usually dominant) means that large numbers of bound-bound transitions need to be taken into account when simulating radiative transfer. Moreover, the large expansion velocities blend the transitions together meaning that it is usually very difficult to define a simple continuum or separate out individual lines. As a consequence, the net contribution of bound-bound transitions tends to dominate over any continuum thermalization opacity (see, e.g. Pinto and Eastman 2000) and fluorescent frequency redistribution becomes key to understanding the overall spectral energy distribution.

The large expansion velocities (and associated velocity gradients) have a central role in shaping the spectral features of supernovae and need to be 
considered in any method that aims to predict synthetic spectra. Several contemporary radiative transfer approaches embed the assumption of highvelocity gradients in the form of the Sobolev approximation (see e.g. Sobolev 1960, Lamers and Casinelli 1999)). This approach makes it relatively easy to take into account very large numbers of bound-bound transitions at modest computational cost (either directly, e.g. Mazzali and Lucy 1993, or via an expansion opacity formalism, e.g. Karp et al. 1977, Blinnikov et al. 1998). However, the Sobolev approximation does have limitations, particularly in relation to the treatment of overlapping lines (e.g. Baron et al. 1996)), which becomes increasingly common at short wavelengths. Consequently, the most sophisticated radiative transfer codes avoid this approximation and treat individual line profiles in detail.

Reasonably accurate radiative transfer simulations also depend strongly on the calculation of the temperatures and ionization/excitation conditions in the ejecta. Local thermodynamic equilibrium (LTE) is often adopted as a first estimate but departures from equilibrium have important consequences and non-LTE effects become increasingly important with time as the ejecta expand. As illustrated by Dessart et al. (2014), accurate synthetic observables depend on describing a range of complicated microphysics, whose role evolves with time. By the latest epochs commonly observed for thermonuclear supernovae ( $\sim 200-300$ days post explosion), the ejecta are very far from LTE: at these "nebular" epochs, ionization and heating controlled by the ongoing injection of non-thermal particles (from radioactive decay) and cooling dominated by multiplets of forbidden lines, predominantly of the iron group elements (for a recent review of the modeling of nebular spectra, see Jerkstrand 2017). Consequently, modeling of spectra at these epochs is critically dependent on the microphysics and quality/quantity of the atomic data (radiative and collisional) available for the necessary ions.

\subsection{Implementation and application to modern explosion models}

Given the competing demands on implementations and computational resources (i.e. need to address complicated/time-dependent microphysics in expanding, inhomogeneous 3D ejecta models), most published studies to date have made several necessary approximations. Currently, perhaps the most important trade-off made in relation to the study of hydrodynamical explosion models is that between simplified microphysics and multidimensional effects in the ejecta. For example, while several of the Monte Carlo radiative transfer codes (e.g. SEDONA, Kasen et al. 2006, or ARTIS, Kromer and Sim 2009) can compute orientation-dependent synthetic observables for fully 3D ejecta models, these codes use the Sobolev approximation and either use LTE or relatively simple nLTE approximations. In contrast, sophisticated 1D codes can avoid the Sobolev approximation and treat many levels of many ions in full nLTE for SNe Ia explosion models (see, e.g., Höflich et al. 1998, Baron et al. 2006, Blondin et al. 2013). 


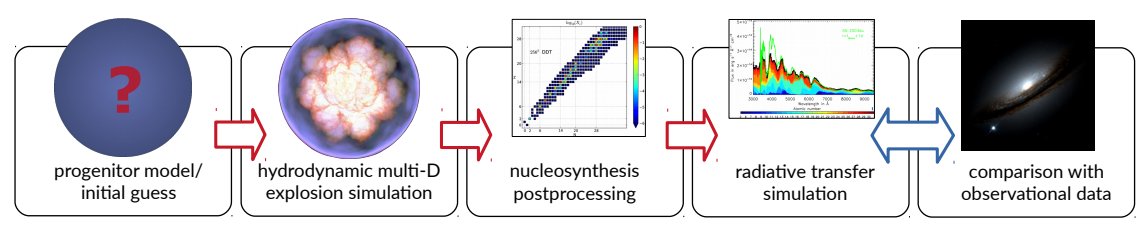

Fig. $13 \mathrm{D}$ modeling pipeline

\section{Scenarios and simulations}

To meet the requirements for a viable explosion scenario discussed in Sect. 2.3 , different modes of ignition and flame propagation are necessary, depending on whether the exploding star is close to the Chandrasekhar-mass or below that limit. The first case constitutes so-called Chandrasekhar-mass explosion models and the second sub-Chandrasekhar mass models. We explore these model classes below. They are addressed with our modeling pipeline shown in Fig. 1 .

\subsection{Chandrasekhar-mass white dwarf explosion models}

Approaching the Chandrasekhar mass, the density in the core of a white dwarf increases steadily. This will lead to the ignition of carbon fusion in the so-called intermediate thermopycnonuclear regime Gasques et al. $2005)$, i.e. under conditions where the density has an appreciable effect on the reaction rate. Initially, energy losses due to neutrinos formed in plasmon decays and electron-nucleus bremsstrahlung cool the stellar center. Ignition occurs when the central density reaches high enough values so that neutrino losses become insufficient to balance the energy production due to carbon burning.

This does, however, not yet trigger the explosion process. It rather leads to a century of "simmering", in which convective motions efficiently transport the energy generated in the stellar center outward. The fluid motions are highly turbulent. On this background, a hotspot finally develops, out of which a deflagration wave is formed by thermonuclear runaway. Simulating the entire simmering phase is virtually impossible, because a century cannot be bridged and the spatial resolution is far from resolving turbulence. Nonetheless, ignition simulations have been performed (Höflich and Stein 2002, Kuhlen et al. 2006, Zingale et al. 2009, Nonaka et al. 2012). A threedimensional simulation following the last hours until the first thermonuclear runaway occurs at a radius of $\sim 50 \mathrm{~km}$ off-center is presented by Nonaka et al. (2012). The results indicate that a second runaway at a different location shortly after the first is unlikely. Thus, to current best knowledge, the deflagration will form in a single region off-center of the WD star. 


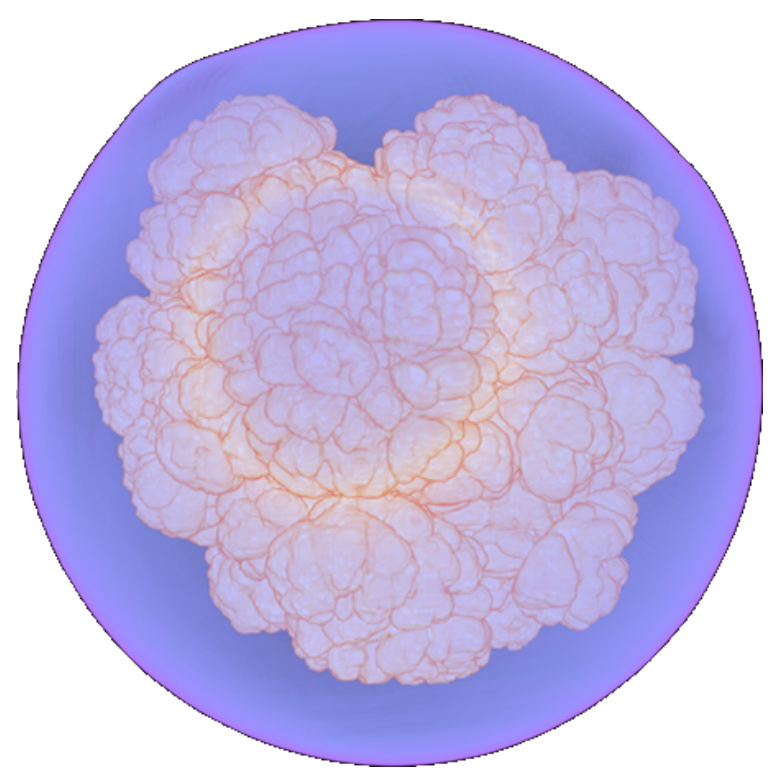

Fig. 2 Simulation of a deflagration (orange/white contour) in a Chandrasekharmass white dwarf star (blue color).

\subsubsection{Pure deflagrations}

The pure deflagration scenario follows possibility (1) described in Sect. 2.3 . After formation of the deflagration wave near the center, it propagates towards the surface, subject to buoyancy instability. Multidimensional simulations clearly show the formation of "mushroom-shaped" bubbles giving rise to a complex morphology of the flame front (see Fig. 2 for an illustration). The flame strongly accelerates due to the interaction with turbulence. The scaling behavior of turbulent motions in this situation was unclear for a long time. Based on highly resolved three-dimensional simulations, however, it could be shown that at small length scales and for most of the burning turbulence is isotropic and follows Kolmogorov scaling (Zingale et al. 2005. Ciaraldi-Schoolmann et al. 2009).

The strength of the deflagration and the overall outcome of the explosion phase fundamentally depend on the initial conditions. Several parameters are expected to vary in nature from event to event, such as the central density and the chemical composition of the exploding white dwarf star. Other parameters are unknown or subject to uncertainties in the numerical modeling. A parameter of models of the explosion phase is the ignition geometry. The length scales of the actual flame formation cannot be resolved in multidimensional simulations. Therefore, the effect is usually mimicked by placing a number of flame kernels near the stellar center. Although this does not necessarily capture the ignition physics, it is a way of defining a well-posed initial setup. For a single sphere, the Rayleigh-Taylor instability is seeded by random numerical noise and imprinting a certain spectrum of 
resolved perturbations on the flame geometry ensures convergence of the model. The simulations of Fink et al. (2014) showed that the initial flame shape has a tremendous impact on the strength of the burning. In this study, a sequence of models was presented with varying numbers of ignition sparks, that were randomly placed in the central region of the white dwarf star. Sparse ignitions naturally lead to aspherical flame geometry evolution, whereas an on average isotropic flame propagation is only possible with dense ignitions. In the former case, only a small fraction of the star is burned. Due to buoyancy, the flame quickly rises towards the surface leaving the far side of the star unaffected. The energy liberated in this process leads to the ejection of parts of the stellar material, and a bound remnant is left behind. In contrast, a complete disruption of the white dwarf star is possible for dense isotropic ignition configurations. In all cases, however, the ejecta structure is well-mixed on large scales due to the flame instabilities. Even with dense ignition configurations, the production of ${ }^{56} \mathrm{Ni}$ hardly exceeds $0.35 M_{\odot}$.

The relatively low ${ }^{56} \mathrm{Ni}$ production means that pure deflagration models generally fail to account for the observed brightness of the majority of SNe Ia (the predicted peak luminosity of such models is too low). However, the ${ }^{56} \mathrm{Ni}$ masses are consistent with those required for some low-luminosity events, of which several sub-classes have now been identified (Taubenberger 2017). In particular, the range of ${ }^{56} \mathrm{Ni}$ masses predicted in pure deflagration models (Jordan et al. 2012b, Fink et al. 2014) is roughly consistent with the observed range of brightness spanned by the Type Iax supernovae (Foley et al. 2013$)$.

The potential identification of Type Iax supernovae with pure deflagrations is supported by comparison of synthetic spectra and light curves to observations. For example, Kromer et al. (2013) compared model predictions for one of the pure deflagration models of Fink et al. (2014) for a range of photospheric-phase epochs to SN2005hk (Phillips et al. 2007), a well-observed member of the SNe Iax class. They found fair agreement in both the strengths and shapes of spectral features across a range of phases in the optical and infrared, and also good correspondence between the model and observed colors around maximum lights. However, some important discrepancies do remain. In particular, the model light curves evolve too quickly, most notably in the post-maximum decline phase in the redder bands (Kromer et al. 2013). This systematic discrepancy is also apparent in the comparison of a different model from the Fink et al. (2014) sample to a fainter member of the SNe Ia class, 2015H (Magee et al.|2016). Several studies have also drawn attention to evidence that the ejecta of SNe Iax are not fully mixed (Stritzinger et al. 2015, Barna et al. 2017), which is difficult to reconcile with a turbulent deflagration model. In addition, it remains unclear whether pure deflagration models can account for the lowest luminosity members of the SNe Iax class, such as SN2008ha (Foley et al. 2009), which requires less than $0.01 M_{\odot}$ of ${ }^{56} \mathrm{Ni}$. Such a low mass of ${ }^{56} \mathrm{Ni}$ might be achieved under conditions whereby burning in the deflagration is occurs only in a limited central region of the $\mathrm{WD}$, for example due to 
an exotic composition (Kromer et al. 2015) - however, it remains to be demonstrated whether this can be realized in nature.

One outstanding, but noteworthy, feature of the comparison of SNe Iax and pure deflagration models is potential role of the residual material from the WD, that was still bound at the end of the explosion phase (in e.g. the models compared to observations mentioned above, $\sim 1 M_{\odot}$ or more of the mass of the initial WD remains bound at the end of the explosion simulation). Some of the ${ }^{56} \mathrm{Ni}$ synthesized in the explosion remains in this material (Kromer et al. 2013, Fink et al. 2014), meaning that it will experience ongoing energy injection which will plausible drive further expulsion of mass (Foley et al. 2016). Further study, both of physical conditions in the residual material (Shen et al. 2017) and of late-phase observations of SNe Iax (Foley et al. 2016) are needed to explore this topic in more detail.

\subsubsection{Delayed detonations}

Enhancing the ${ }^{56} \mathrm{Ni}$ production and the explosion energy to values necessary to reproduce normal SNe Ia is not possible by simply increasing the number of ignition kernels or tuning the initial parameters of the exploding white dwarf. A fundamental change in the burning mode is required - a transition from the initial deflagration, that is necessary to pre-expand the material, to a subsequent detonation. This scenario follows possibility (2) described in Sect. 2.3 This is the idea of the class of delayed detonation models. The key question in these is obviously if and how a detonation is triggered in a late burning stage.

Several mechanisms have been proposed for initiating detonations in delayed detonation models. A spontaneous deflagration-to-detonation transition (DDT) may be caused by intrinsic processes in the burning wave. Whether or not such DDTs occur in thermonuclear supernova explosions remains uncertain. Some necessary conditions were laid out in the studies of (Lisewski et al. 2000b, Woosley 2007, Woosley et al. 2009, 2011). Two other mechanisms, the gravitationally confined detonation (GCD, Plewa et al. 2004) and the pulsational delayed detonation (PDD) mechanisms (e.g., Bravo and García-Senz 2006), rely on weak initial deflagration stages that fail to gravitationally unbind the white dwarf star.

The mechanism for GCD assumes that a one-sided ignition leads to an asymmetric deflagration that is too weak to gravitationally unbind the WD star. The deflagration ash breaks out of the star's surface and sweeps around it to collide in the antipode. Clearly, a successful ignition of a detonation in this collision favors stronger impact which in turn implies a weak deflagration phase. The resulting detonation then burns the bound core of the object. With weak deflagrations, it will not be very expanded and thus particularly bright events with high masses of synthesized ${ }^{56} \mathrm{Ni}$ are expected. A bound white dwarf resulting from a weak deflagration will pulsate. These pulsations may aid the formation of a detonation (Jordan et al. 2012a). Both the GCD and the PDD scenarios share the characteristic feature that the products of high-density deflagration will be located in the outer part of 
the ejected material at high velocities. This is in conflict with observations (Seitenzahl et al. 2016).

Also for the classical (DDT) delayed detonation scenario, several problems persist. It has been suggested as a model for the bulk of normal SNe Ia. This requires them to reproduce individual supernova observations. Studies based on 1D DDT models have generally been fairly successful in this regard (e.g. Höflich et al. 1998, Blondin et al. 2013). Radiative transfer simulations based on multi-dimensional simulations of DDTs (e.g., in 2D Kasen et al. 2009, Blondin et al. 2011, or in 3D Seitenzahl et al. 2013, Sim et al. 2013) have also generally found that some DDT models can provide a fairly good (albeit far from perfect) match to many properties of the light curves spectra, and indeed spectropolarimetry (Bulla et al. 2016) of individual SNe Ia.

In addition, if DDT models are responsible for the full population, observed trends between characteristic features should be reproduced. The model explosions should be able to cover the range of brightnesses observed from normal SNe Ia. This requires a nickel mass production in the range from below $0.4 M_{\odot}$ to $0.8 M_{\odot}$, see e.g. Scalzo et al. (2014). Delayed detonations face a fundamental challenge here. Generally, stronger deflagrations lead to increased expansion before the detonation phase sets in (Röpke and Niemeyer 2007, Mazzali et al. 2007). Consequently, the detonation runs over lower-density material and produces less ${ }^{56} \mathrm{Ni}$. Therefore, the faintest models are expected to be those with the strongest deflagration. This was tested in multi-spot ignition setups that allow to vary the deflagration strength significantly. The strongest deflagrations produce $\gtrsim 0.3 M_{\odot}$ of ${ }^{56} \mathrm{Ni}$ and in the subsequent detonation little is added to this amount. This means that multi-spot ignitions with many, on average isotropically distributed kernels are required to reach the fainter end of the distribution of normal SNe Ia. These, however seem unlikely to be realized in nature (Nonaka et al. 2012). Furthermore, when such models are invoked, they appear to fail to fully produce the relatively rapid light curve evolution that is observed to coincide with low luminosity (i.e. the light curve width-luminosity relation: see e.g. Sim et al. $(2013))$. The second problem in this context is that the brightness distribution of normal events is observed to peak at explosions producing $\sim 0.6 M_{\odot}$ of ${ }^{56} \mathrm{Ni}$. It is not obvious why the initial parameters such as flame ignition geometry, central density and chemical composition of the progenitor white dwarf star, or the initiation mechanism of the detonation, should favor a configuration producing this amount of radioactive nickel.

We note that, although the 3D simulations of Sim et al. (2013) have difficulties reproducing the observed width-luminosity relation with a faster decline of the $B$-band light curve for fainter events, it may be possible to construct such a relation in Chandrasekhar-mass white dwarf star explosions (Kasen and Woosley 2007). Recent studies (Blondin et al. 2017, Goldstein and Kasen 2018), however, increasingly indicate that sub-Chandrasekhar mass explosions are required to capture the full range of the observed relation. 
4.2 Sub-Chandrasekhar mass white dwarf explosions

The alternative to Chandrasekhar-mass white dwarf explosions as models for SNe Ia are detonations in sub-Chandrasekhar mass objects following possibility (3) of Sect. 2.3. The capability of the scenario to reproduce basic characteristics of observed SNe Ia was demonstrated by sequences of toy models. Here, if white dwarfs of varying mass are set up and a central detonation ignited artificially, the resulting ejecta structures provide a relatively simple sequence of models that do a fair job of reproducing many of the observed characteristics of SNe Ia (e.g. Shigeyama et al. 1992, Sim et al. 2010, Shen et al. 2017). A very attractive feature of this class of model is that a single simple physical parameter can be recognized as responsible for driving many of the differences between different explosions: the mass of the exploding WD. In particular, a wide range of nickel mass can be produced from only a modest range of WD masses: an initial WD mass of around $1 \mathrm{M}_{\odot}$ is required to produce an explosion with brightness characteristic of the most common SNe Ia (see Sim et al. 2010, Shen et al. 2017). In addition, the low densities in sub-Chandrasekhar mass white dwarfs means that their detonation yields significant quantities of intermediate mass elements, allowing for a relatively good match to observed spectra across a broad range of explosion luminosity. One important challenge for this class of simple (toy) sub-Chandrasekhar mass models is that they struggle to sustain sufficiently slow light curve evolution to account for the brighter end of the SNe Ia distribution. However, the variation in ejecta mass amongst sub-Chandrasekhar mass models does naturally suggest a link between brightness and light curve evolution and simulations have favored sub-Chandrasekhar models for relatively faint explosions (see, e.g., Blondin et al. 2017).

The problem with sub-Chandrasekhar mass explosion models of the sort described above is that the physical ignition of detonations in such objects does not intrinsically arise from an evolutionary process (such as the accretion of mass towards the Chandrasekhar limit). It has to be caused by some vigorous event. Two possibilities are commonly discussed.

One way to ignite a detonation in a carbon-oxygen white dwarf star is that it accretes helium-rich material from a companion. Due to instabilities in the accretion process or once the accreted shell has grown massive enough, a detonation triggers in the He material. It propagates the carbonoxygen core and drives a shock wave into it. This shock wave may trigger a secondary detonation in carbon-oxygen rich material - constituting the so-called double detonation explosion scenario (see Fink et al. 2010, Moll and Woosley 2013 for recent multidimensional supernova simulations following this paradigm). It is conceivable that this occurs when the shock hits the outer edge of the core ("edge-lit double detonation") or due to a spherical collimation of the inwards propagating oblique shock wave near its center. The latter case was shown to robustly lead to detonations of the core by a geometric amplification effect (Fink et al. 2007). It has to be emphasized, however, that many of the published models simply assume a primary detonation of the He shell. The ignition process is very hard to 
resolve numerically and the success of the scenario hinges on it to occur in reality. Synthetic spectra have been computed from double detonation models for a range of conditions (e.g. Nugent et al. 1997, Kromer et al. 2010, Woosley and Kasen 2011), with results that depend significantly on the assumed structure and mass of the helium layer at explosion. Indeed, it generally appears to be the case that the influence of the outer ejecta layers (rich in helium and/or helium-detonation ash) is mostly detrimental to the agreement of the models with observations: if substantial helium shells are invoked this leads to effectively suppressing the characteristic features of intermediate mass elements and to very dramatic line blanketing effects associated with heavy elements that are synthesized in the helium detonation. Thus, for such double detonation models to be viable, very low mass helium shells must be invoked: whether such low masses of helium can really be ignited and/or sustain a detonation is a topic of active study (e.g. Shen and Bildsten 2007?, 2014).

An alternative scenario is that of violent mergers (Pakmor et al. 2010. 2011, 2012). In contrast to the classical merger paradigm, the explosion happens before the two white dwarfs have formed a single object. In the inspiral process, the lighter of the pair is disrupted and its material plunges into the more massive primary that is only weakly affected by tidal forces. This impact may trigger a detonation of the primary - a sub-Chandrasekhar mass white dwarf. A recent update of the model (Pakmor et al. 2013) suggests that the ignition of the detonation is triggered even earlier in the inspiral process when He rich material (that always exists in low quantities on top of a carbon-oxygen white dwarf) is accreted from the secondary to the primary. This rapid accretion process leads to hydrodynamical instabilities in the He-layer on the primary and triggers a detonation in this shell. Similar to the double detonation scenario, the actual supernovae results from a secondary detonation of the core material. In contrast to that scenario, however, in the violent merger case the He shell is less massive and less dense so that its imprint on the predicted observables is much reduced.

It is remarkable that population synthesis studies predict a peak of the distribution of white dwarf mergers at primary masses that produce $\sim 0.6 M_{\odot}$ of ${ }^{56} \mathrm{Ni}$ (Ruiter et al. 2013). Moreover, also the temporal evolution of the luminosity function resulting from sub-Chandrasekhar mass WD detonations seems to match the observations (Shen et al. 2017).

\section{Conclusions}

Thanks to advances in computing resources and numerical methods in recent years, we are now able to perform meaningful fully 3D explosion simulations for a range of progenitor scenarios that have been proposed for Type Ia supernovae. Combined with radiative transfer post-processing, which allows predictions to be made that can be directly compared to observations, such simulations are now playing a key role in driving our understanding of the nature and physics of thermonuclear supernovae. However, the state of the art remains incomplete and far from satisfactory - numerous 
limitations persist. These include clearly posing initial conditions for explosion simulations in the context of particular progenitor modeling, proper representation of the dynamics and instabilities during the thermonuclear combustion in full star models, and adequate description of the complex radiation processes responsible for spectrum formation in the evolving ejecta. The last decade has demonstrated that such multi-dimensional simulations are possible. The goal for the future will be their development towards a level of predictive power than allows for ever-improving quantitative testing by comparison to the increasing wealth of observational data.

Acknowledgements The work of FKR is supported by the Klaus Tschira Foundation and by the Collaborative Research Center SFB 881 "The Milky Way System" (subproject A10) of the German Research Foundation (DFG).

\section{References}

B. Barna, T. Szalai, M. Kromer, W.E. Kerzendorf, J. Vinkó, J.M. Silverman, G.H. Marion, J.C. Wheeler, Abundance tomography of Type Iax SN 2011ay with tardis. MNRAS 471, 4865-4877 (2017).

E. Baron, P.H. Hauschildt, A. Mezzacappa, Radiative transfer in the comoving frame. MNRAS 278, 763-772 (1996)

E. Baron, S. Bongard, D. Branch, P.H. Hauschildt, Spectral Modeling of SNe Ia Near Maximum Light: Probing the Characteristics of Hydrodynamical Models. ApJ 645, 480-487 (2006).

S.I. Blinnikov, E.I. Sorokina, Supernova Explosions inside Carbon-Oxygen Circumstellar Shells. ArXiv e-prints (2010)

S.I. Blinnikov, R. Eastman, O.S. Bartunov, V.A. Popolitov, S.E. Woosley, A comparative modeling of supernova 1993J. ApJ 496, 454-472 (1998).

S. Blondin, D. Kasen, F.K. Röpke, R.P. Kirshner, K.S. Mandel, Confronting 2d delayed-detonation models with light curves and spectra of Type Ia supernovae. MNRAS 417, 1280-1302 (2011).

S. Blondin, L. Dessart, D.J. Hillier, A.M. Khokhlov, One-dimensional delayeddetonation models of Type Ia supernovae: confrontation to observations at bolometric maximum. MNRAS 429, 2127-2142 (2013).

S. Blondin, L. Dessart, D.J. Hillier, A.M. Khokhlov, Evidence for subChandrasekhar-mass progenitors of Type Ia supernovae at the faint end of the width-luminosity relation. MNRAS 470, 157-165 (2017).

E. Bravo, D. García-Senz, Beyond the bubble catastrophe of Type Ia supernovae: Pulsating reverse detonation models. ApJ 642, 157-160 (2006).

M. Bulla, S.A. Sim, M. Kromer, I.R. Seitenzahl, M. Fink, F. Ciaraldi-Schoolmann, F.K. Röpke, W. Hillebrandt, R. Pakmor, A.J. Ruiter, S. Taubenberger, Predicting polarization signatures for double-detonation and delayed-detonation models of Type Ia supernovae. MNRAS 462, 1039-1056 (2016).

A.C. Calder, D.M. Townsley, I.R. Seitenzahl, F. Peng, O.E.B. Messer, N. Vladimirova, E.F. Brown, J.W. Truran, D.Q. Lamb, Capturing the Fire: Flame Energetics and Neutronization for Type Ia Supernova Simulations. ApJ 656, 313-332 (2007).

F. Ciaraldi-Schoolmann, W. Schmidt, J.C. Niemeyer, F.K. Röpke, W. Hillebrandt, Turbulence in a three-dimensional deflagration model for Type Ia supernovae. I. Scaling properties. ApJ 696, 1491-1497 (2009).

G. Damköhler, Der Einfluß der Turbulenz auf die Flammengeschwindigkeit in Gasgemischen. Z. f. Elektroch. 46(11), 601-652 (1940)

L. Dessart, D.J. Hillier, S. Blondin, A. Khokhlov, Critical ingredients of Type Ia supernova radiative-transfer modelling. MNRAS 441, 3249-3270 (2014). 
M. Fink, W. Hillebrandt, F.K. Röpke, Double-detonation supernovae of subChandrasekhar mass white dwarfs. A\&A 476, 1133-1143 (2007).

M. Fink, F.K. Röpke, W. Hillebrandt, I.R. Seitenzahl, S.A. Sim, M. Kromer, Double-detonation sub-Chandrasekhar supernovae: can minimum helium shell masses detonate the core? A\&A 514, 53 (2010).

M. Fink, M. Kromer, I.R. Seitenzahl, F. Ciaraldi-Schoolmann, F.K. Röpke, S.A. Sim, R. Pakmor, A.J. Ruiter, W. Hillebrandt, Three-dimensional pure deflagration models with nucleosynthesis and synthetic observables for Type Ia supernovae. MNRAS 438, 1762-1783 (2014).

R.J. Foley, R. Chornock, A.V. Filippenko, M. Ganeshalingam, R.P. Kirshner, W. Li, S.B. Cenko, P.J. Challis, A.S. Friedman, M. Modjaz, J.M. Silverman, W.M. Wood-Vasey, SN 2008ha: An Extremely Low Luminosity and Exceptionally Low Energy Supernova. AJ 138, 376-391 (2009).

R.J. Foley, P.J. Challis, R. Chornock, M. Ganeshalingam, W. Li, G.H. Marion, N.I. Morrell, G. Pignata, M.D. Stritzinger, J.M. Silverman, X. Wang, J.P. Anderson, A.V. Filippenko, W.L. Freedman, M. Hamuy, S.W. Jha, R.P. Kirshner, C. McCully, S.E. Persson, M.M. Phillips, D.E. Reichart, A.M. Soderberg, Type Iax Supernovae: A New Class of Stellar Explosion. ApJ 767, 57 (2013).

R.J. Foley, S.W. Jha, Y.-C. Pan, W.K. Zheng, L. Bildsten, A.V. Filippenko, D. Kasen, Late-time spectroscopy of Type Iax supernovae. MNRAS 461, 433-457 (2016).

C.L. Fryer, A.J. Ruiter, K. Belczynski, P.J. Brown, F. Bufano, S. Diehl, C.J. Fontes, L.H. Frey, S.T. Holland, A.L. Hungerford, S. Immler, P. Mazzali, C. Meakin, P.A. Milne, C. Raskin, F.X. Timmes, Spectra of Type Ia Supernovae from Double Degenerate Mergers. ApJ 725, 296-308 (2010).

L.R. Gasques, A.V. Afanasjev, E.F. Aguilera, M. Beard, L.C. Chamon, P. Ring, M. Wiescher, D.G. Yakovlev, Nuclear fusion in dense matter: Reaction rate and carbon burning. Phys. Rev. C 72(2), 025806 (2005).

D.A. Goldstein, D. Kasen, Evidence for Sub-Chandrasekhar Mass Type Ia Supernovae from an Extensive Survey of Radiative Transfer Models. ApJ 852, 33 (2018).

E.P. Hicks, R. Rosner, Gravitationally unstable flames: Rayleigh-taylor stretching versus turbulent wrinkling. ApJ 771, 135 (2013).

P. Höflich, J. Stein, On the thermonuclear runaway in Type Ia supernovae: How to run away? ApJ 568, 779-790 (2002).

P. Höflich, J.C. Wheeler, F.K. Thielemann, Type Ia supernovae: Influence of the initial composition on the nucleosynthesis, light curves, and spectra and consequences for the determination of omega $m$ and lambda. ApJ 495, 617-629 (1998).

B. Hristov, D.C. Collins, P. Hoeflich, C.A. Weatherford, T.R. Diamond, Magnetohydrodynamical effects on nuclear deflagration fronts in Type Ia supernovae. ArXiv e-prints (2017)

A.P. Jackson, D.M. Townsley, A.C. Calder, Power-law wrinkling turbulence-flame interaction model for astrophysical flames. ApJ 784, 174 (2014).

A. Jerkstrand, Spectra of supernovae in the nebular phase, in Handbook of Supernovae, ed. by A. Alsabti, P. Murdin (Springer, ???, 2017), pp. 795-842

G.C. Jordan IV, C. Graziani, R.T. Fisher, D.M. Townsley, C. Meakin, K. Weide, L.B. Reid, J. Norris, R. Hudson, D.Q. Lamb, The detonation mechanism of the pulsationally assisted gravitationally confined detonation model of type Ia supernovae. ApJ 759, 53 (2012a).

G.C. Jordan IV, H.B. Perets, R.T. Fisher, D.R. van Rossum, Failed-detonation Supernovae: Subluminous Low-velocity Ia Supernovae and their Kicked Remnant White Dwarfs with Iron-rich Cores. ApJ 761, 23 (2012b).

A.H. Karp, G. Lasher, K.L. Chan, E.E. Salpeter, The opacity of expanding media - The effect of spectral lines. ApJ 214, 161-178 (1977).

D. Kasen, S.E. Woosley, On the origin of the Type Ia supernova width-luminosity relation. ApJ 656, 661-665 (2007).

D. Kasen, F.K. Röpke, S.E. Woosley, The diversity of type Ia supernovae from broken symmetries. Nature 460, 869-872 (2009). 
D. Kasen, R.C. Thomas, P. Nugent, Time-dependent monte carlo radiative transfer calculations for three-dimensional supernova spectra, light curves, and polarization. ApJ 651, 366-380 (2006).

A.M. Khokhlov, Propagation of turbulent flames in supernovae. ApJ 449, 695-713 (1995).

A.M. Khokhlov, E.S. Oran, J.C. Wheeler, Deflagration-to-detonation transition in thermonuclear supernovae. ApJ 478, 678-688 (1997).

M. Kromer, S.A. Sim, Time-dependent three-dimensional spectrum synthesis for Type Ia supernovae. MNRAS 398, 1809-1826 (2009).

M. Kromer, S.A. Sim, M. Fink, F.K. Röpke, I.R. Seitenzahl, W. Hillebrandt, Double-detonation Sub-Chandrasekhar Supernovae: Synthetic Observables for Minimum Helium Shell Mass Models. ApJ 719, 1067-1082 (2010).

M. Kromer, M. Fink, V. Stanishev, S. Taubenberger, F. Ciaraldi-Schoolman, R. Pakmor, F.K. Röpke, A.J. Ruiter, I.R. Seitenzahl, S.A. Sim, G. Blanc, N. EliasRosa, W. Hillebrandt, 3D deflagration simulations leaving bound remnants: a model for 2002cx-like Type Ia supernovae. MNRAS 429, 2287-2297 (2013).

M. Kromer, S.T. Ohlmann, R. Pakmor, A.J. Ruiter, W. Hillebrandt, K.S. Marquardt, F.K. Röpke, I.R. Seitenzahl, S.A. Sim, S. Taubenberger, Deflagrations in hybrid CONe white dwarfs: a route to explain the faint Type Iax supernova 2008ha. MNRAS 450, 3045-3053 (2015).

M. Kuhlen, S.E. Woosley, G.A. Glatzmaier, Carbon ignition in Type Ia supernovae. II. A three-dimensional numerical model. ApJ 640, 407-416 (2006).

H.J.G.L.M. Lamers, J.P. Casinelli, Introduction to Stellar Winds (Cambridge University Press, Cambridge, ???, 1999)

A.M. Lisewski, W. Hillebrandt, S.E. Woosley, Constraints on the delayed transition to detonation in Type Ia supernovae. ApJ 538, 831-836 (2000a).

A.M. Lisewski, W. Hillebrandt, S.E. Woosley, J.C. Niemeyer, A.R. Kerstein, Distributed burning in Type Ia supernovae: A statistical approach. ApJ 537, 405$413(2000 \mathrm{~b})$.

M.R. Magee, R. Kotak, S.A. Sim, M. Kromer, D. Rabinowitz, S.J. Smartt, C. Baltay, H.C. Campbell, T.-W. Chen, M. Fink, A. Gal-Yam, L. Galbany, W. Hillebrandt, C. Inserra, E. Kankare, L. Le Guillou, J.D. Lyman, K. Maguire, R. Pakmor, F.K. Röpke, A.J. Ruiter, I.R. Seitenzahl, M. Sullivan, S. Valenti, D.R. Young, The type Iax supernova, SN 2015H. A white dwarf deflagration candidate. A\&A 589, 89 (2016).

P.A. Mazzali, L.B. Lucy, The application of Monte Carlo methods to the synthesis of early-time supernovae spectra. A\&A 279, 447-456 (1993).

P.A. Mazzali, F.K. Röpke, S. Benetti, W. Hillebrandt, A common explosion mechanism for Type Ia supernovae. Science 315, 825-828 (2007).

R. Moll, S.E. Woosley, Multi-dimensional Models for Double Detonation in SubChandrasekhar Mass White Dwarfs. ApJ 774, 137 (2013)

J.C. Niemeyer, W. Hillebrandt, Turbulent nuclear flames in type Ia supernovae. ApJ 452, 769-778 (1995).

U.M. Noebauer, S. Taubenberger, S. Blinnikov, E. Sorokina, W. Hillebrandt, Type Ia supernovae within dense carbon- and oxygen-rich envelopes: a model for 'Super-Chandrasekhar' explosions? MNRAS 463, 2972-2985 (2016).

A. Nonaka, A.J. Aspden, M. Zingale, A.S. Almgren, J.B. Bell, S.E. Woosley, Highresolution simulations of convection preceding ignition in Type Ia supernovae using adaptive mesh refinement. ApJ 745, 73 (2012).

P. Nugent, E. Baron, D. Branch, A. Fisher, P.H. Hauschildt, Synthetic Spectra of Hydrodynamic Models of Type IA Supernovae. ApJ 485, 812-819 (1997).

S. Osher, J.A. Sethian, Fronts propagating with curvature-dependent speed: Algorithms based on Hamilton-Jacobi formulations. Journal of Computational Physics 79, 12-49 (1988)

R. Pakmor, M. Kromer, F.K. Röpke, S.A. Sim, A.J. Ruiter, W. Hillebrandt, Subluminous type ia supernovae from the mergers of equal-mass white dwarfs with mass $\sim 0.9 \mathrm{~m} \odot$. Nature 463, 61-64 (2010).

R. Pakmor, S. Hachinger, F.K. Röpke, W. Hillebrandt, Violent mergers of nearly equal-mass white dwarf as progenitors of subluminous Type Ia supernovae. 
A\&A 528, 117 (2011).

R. Pakmor, M. Kromer, S. Taubenberger, S.A. Sim, F.K. Röpke, W. Hillebrandt, Normal Type Ia supernovae from violent mergers of white dwarf binaries. ApJ 747, 10 (2012).

R. Pakmor, M. Kromer, S. Taubenberger, V. Springel, Helium-ignited violent mergers as a unified model for normal and rapidly declining type Ia supernovae. ApJ 770, 8 (2013).

M.M. Phillips, W. Li, J.A. Frieman, S.I. Blinnikov, D. DePoy, J.L. Prieto, P. Milne, C. Contreras, G. Folatelli, N. Morrell, M. Hamuy, N.B. Suntzeff, M. Roth, S. González, W. Krzeminski, A.V. Filippenko, W.L. Freedman, R. Chornock, S. Jha, B.F. Madore, S.E. Persson, C.R. Burns, P. Wyatt, D. Murphy, R.J. Foley, M. Ganeshalingam, F.J.D. Serduke, K. Krisciunas, B. Bassett, A. Becker, B. Dilday, J. Eastman, P.M. Garnavich, J. Holtzman, R. Kessler, H. Lampeitl, J. Marriner, S. Frank, J.L. Marshall, G. Miknaitis, M. Sako, D.P. Schneider, K. van der Heyden, N. Yasuda, The peculiar SN 2005hk: Do some Type Ia supernovae explode as deflagrations? PASP 119, 360-387 (2007).

P.A. Pinto, R.G. Eastman, The physics of Type Ia supernova light curves. II. Opacity and diffusion. ApJ 530, 757-776 (2000).

T. Plewa, A.C. Calder, D.Q. Lamb, Type Ia supernova explosion: Gravitationally confined detonation. ApJ 612, 37-40 (2004).

A.Y. Poludnenko, T.A. Gardiner, E.S. Oran, Spontaneous Transition of Turbulent Flames to Detonations in Unconfined Media. Physical Review Letters 107(5), 054501 (2011).

M. Reinecke, W. Hillebrandt, J.C. Niemeyer, R. Klein, A. Gröbl, A new model for deflagration fronts in reactive fluids. A\&A 347, 724-733 (1999)

F.K. Röpke, Combustion in thermonuclear supernova explosions, in Handbook of Supernovae, ed. by A. Alsabti, P. Murdin (Springer, ???, 2017), pp. 1185-1209

F.K. Röpke, J.C. Niemeyer, Delayed detonations in full-star models of type Ia supernova explosions. A\&A 464, 683-686 (2007).

F.K. Röpke, W. Schmidt, Turbulent combustion in thermonuclear supernovae, in Interdisciplinary Aspects of Turbulence, ed. by W. Hillebrandt, F. Kupka Lecture Notes in Physics (Springer, Berlin, 2009), pp. 255-289.

F.K. Röpke, S.E. Woosley, W. Hillebrandt, Off-center ignition in Type Ia supernovae. I. initial evolution and implications for delayed detonation. ApJ 660, 1344-1356 (2007).

A.J. Ruiter, S.A. Sim, R. Pakmor, M. Kromer, I.R. Seitenzahl, K. Belczynski, M. Fink, M. Herzog, W. Hillebrandt, F.K. Röpke, S. Taubenberger, On the brightness distribution of Type Ia supernovae from violent white dwarf mergers. MNRAS 429, 1425-1436 (2013).

R. Scalzo, G. Aldering, P. Antilogus, C. Aragon, S. Bailey, C. Baltay, S. Bongard, C. Buton, F. Cellier-Holzem, M. Childress, N. Chotard, Y. Copin, H.K. Fakhouri, E. Gangler, J. Guy, A.G. Kim, M. Kowalski, M. Kromer, J. Nordin, P. Nugent, K. Paech, R. Pain, E. Pecontal, R. Pereira, S. Perlmutter, D. Rabinowitz, M. Rigault, K. Runge, C. Saunders, S.A. Sim, G. Smadja, C. Tao, S. Taubenberger, R.C. Thomas, B.A. Weaver, Nearby Supernova Factory, Type Ia supernova bolometric light curves and ejected mass estimates from the Nearby Supernova Factory. MNRAS 440, 1498-1518 (2014).

W. Schmidt, J.C. Niemeyer, W. Hillebrandt, F.K. Röpke, A localised subgrid scale model for fluid dynamical simulations in astrophysics. II. Application to type Ia supernovae. A\&A 450, 283-294 (2006).

W. Schmidt, F. Ciaraldi-Schoolmann, J.C. Niemeyer, F.K. Röpke, W. Hillebrandt, Turbulence in a three-dimensional deflagration model for Type Ia supernovae. II. Intermittency and the deflagration-to-detonation transition probability. ApJ 710, 1683-1693 (2010).

I.R. Seitenzahl, F. Ciaraldi-Schoolmann, F.K. Röpke, M. Fink, W. Hillebrandt, M. Kromer, R. Pakmor, A.J. Ruiter, S.A. Sim, S. Taubenberger, Three-dimensional delayed-detonation models with nucleosynthesis for Type Ia supernovae. MNRAS 429, 1156-1172 (2013).

I.R. Seitenzahl, M. Kromer, S.T. Ohlmann, F. Ciaraldi-Schoolmann, K. Marquardt, 
M. Fink, W. Hillebrandt, R. Pakmor, F.K. Röpke, A.J. Ruiter, S.A. Sim, S. Taubenberger, Three-dimensional simulations of gravitationally confined detonations compared to observations of SN 1991T. A\&A 592, 57 (2016).

G.J. Sharpe, The structure of steady detonation waves in Type Ia supernovae: pathological detonations in C-O cores. MNRAS 310, 1039-1052 (1999).

K.J. Shen, L. Bildsten, Thermally stable nuclear burning on accreting white dwarfs. ApJ 660, 1444-1450 (2007).

K.J. Shen, L. Bildsten, The Ignition of Carbon Detonations via Converging Shock Waves in White Dwarfs. ApJ 785, 61 (2014).

K.J. Shen, S. Toonen, O. Graur, The evolution of the Type Ia supernova luminosity function. ApJ 851, 50 (2017).

T. Shigeyama, K. Nomoto, H. Yamaoka, F. Thielemann, Possible models for the type IA supernova 1990N. ApJ 386, 13-16 (1992).

S.A. Sim, F.K. Röpke, W. Hillebrandt, M. Kromer, R. Pakmor, M. Fink, A.J. Ruiter, I.R. Seitenzahl, Detonations in sub-Chandrasekhar-mass $\mathrm{C}+\mathrm{O}$ white dwarfs. ApJ 714, 52-57 (2010).

S.A. Sim, I.R. Seitenzahl, M. Kromer, F. Ciaraldi-Schoolmann, F.K. Röpke, M. Fink, W. Hillebrandt, R. Pakmor, A.J. Ruiter, S. Taubenberger, Synthetic light curves and spectra for three-dimensional delayed-detonation models of type ia supernovae. MNRAS 436, 333-347 (2013).

V.V. Sobolev, Moving envelopes of stars (Cambridge: Harvard University Press, ???, 1960)

M.D. Stritzinger, S. Valenti, P. Hoeflich, E. Baron, M.M. Phillips, F. Taddia, R.J. Foley, E.Y. Hsiao, S.W. Jha, C. McCully, V. Pandya, J.D. Simon, S. Benetti, P.J. Brown, C.R. Burns, A. Campillay, C. Contreras, F. Förster, S. Holmbo, G.H. Marion, N. Morrell, G. Pignata, Comprehensive observations of the bright and energetic Type Iax SN 2012Z: Interpretation as a Chandrasekhar mass white dwarf explosion. A\&A 573, 2 (2015).

S. Taubenberger, The extremes of thermonuclear supernovae, 317-373 (2017)

F.X. Timmes, S.E. Woosley, The conductive propagation of nuclear flames. I. degenerate $\mathrm{C}+\mathrm{O}$ and $\mathrm{O}+\mathrm{Ne}+\mathrm{Mg}$ white dwarfs. ApJ 396, 649-667 (1992).

C. Travaglio, W. Hillebrandt, M. Reinecke, F.-K. Thielemann, Nucleosynthesis in multi-dimensional SN Ia explosions. A\&A 425, 1029-1040 (2004).

N. Vladimirova, G.V. Weirs, L. Ryzhik, Flame capturing with an advectionreaction-diffusion model. Combustion Theory Modelling 10(5), 727-747 (2006).

S.E. Woosley, Type Ia supernovae: Burning and detonation in the distributed regime. ApJ 668, 1109-1117 (2007).

S.E. Woosley, D. Kasen, Sub-Chandrasekhar Mass Models for Supernovae. ApJ 734, 38 (2011).

S.E. Woosley, A.R. Kerstein, A.J. Aspden, Flames in type Ia supernova: Deflagration-detonation transition in the oxygen-burning flame. ApJ 734, 37 (2011).

S.E. Woosley, D. Kasen, S. Blinnikov, E. Sorokina, Type Ia supernova light curves. ApJ 662, 487-503 (2007)

S.E. Woosley, A.R. Kerstein, V. Sankaran, A.J. Aspden, F.K. Röpke, Type Ia Supernovae: Calculations of Turbulent Flames Using the Linear Eddy Model. ApJ 704, 255-273 (2009).

Y.B. Zel'dovich, V.B. Librovich, G.M. Makhviladze, G.I. Sivashinskii, On the onset of detonation in a nonuniformly heated gas. Journal of Applied Mechanics and Technical Physics 11, 264-270 (1970).

M. Zingale, S.E. Woosley, C.A. Rendleman, M.S. Day, J.B. Bell, Three-dimensional numerical simulations of Rayleigh-Taylor unstable flames in Type Ia supernovae. ApJ 632, 1021-1034 (2005).

M. Zingale, A.S. Almgren, J.B. Bell, A. Nonaka, S.E. Woosley, Low Mach Number Modeling of Type IA Supernovae. IV. White Dwarf Convection. ApJ 704, 196$210(2009)$. 